ANNALES

UNIVERSITATIS MARIAE CURIE-SKŁODOWSKA

LUBLIN - POLONIA

VOL. LXXII, 1

SECTIO AA

2017

\title{
Stability of colloidal systems - a review of the stability measurements methods
}

\author{
Jakub Matusiak ${ }^{*}$ and Elżbieta Grządka \\ Maria Curie-Sklodowska University, Faculty of Chemistry, \\ Department of Radiochemistry and Colloid Chemistry, \\ M. Curie-Sklodowska Sq. 3, 20-031 Lublin, Poland \\ e-mail: jcb.matusiak@gmail.com
}

The stability of colloidal systems is very important in numerous already existing and new formulations. In most cases if such systems are not characterized by an appropriate stability they can not find any useful applications. The opposite process to the stabilization is the flocculation. Generally, it is undesirable. However, in a few cases the flocculation is very useful, for example in the wastewater treatment. That is why the methods used to determine stabilizing-flocculating properties of the colloidal systems are of significant importance.

The paper describes types of stability and flocculation as well as the factors influencing those processes, e.g. the addition of polymers or surfactants. The methods presented in this paper are UV-VIS spectrophotometry, turbidimetry, zeta potential and density measurements.

Keywords: stability, colloids, zeta potential, turbidimetry, spectroscopy

\section{INTRODUCTION}

The continuous growth of our society requires developing some new products and methods for their analysis. Colloidal systems are very common in everyday life. Such systems are the basis of many already 
existing formulations. It is hard to imagine a product that is not a colloid. For example, almost every cosmetic used nowadays is an emulsion. Besides, colloidal systems are used in the production of food and beverages, medicines, paints and others [1-2]. Referring to the abovementioned fact, the concept of colloidal systems stability is very important. Colloids contain two phases in which one substance is dispersed in the other. The dispersed substance is usually called the dispersed phase, and the dispersant is also called a continuous phase. Due to the fact that colloidal systems are usually not true solutions but a homogenous mixture of two or more substances, they are not stable in time [3]. After some time, the dispersed phase could precipitate from the continuous phase in the form of flocks. In this case phase separation occurs, which influences the properties of the system. Therefore it is very important to maintenance the stability of colloidal systems. There are different types of stability such as: kinetic, thermodynamic, electrostatic and steric (depletion). The kinetic stability is associated with the existence of energy barrier that prevents the particles from coagulation. The thermodynamic stability refers to the situation in which the coagulated phase has higher free energy than the dispersed one. The electrostatic stabilization is the consequence of the electrostatic repulsion forces. If the particles suspended in the liquid medium possess the same electrostatic charge on its surface they do not attract each other which results in the stability increase [8]. However, this type of stability applies only to the systems in which the range of the electrostatic repulsion is higher than that of the attractive forces and to those containing small particles. Electrostatic stabilization is considered to be short-termed [9]. The last type of stability is steric which is obtained by the addition of macromolecules to the sytem. As it was mentioned before stability can be increased by the addition of surfactants or polymers to the systems which influences the process of stability by changing the interphase properties [4-7].The above-mentioned substances can adsorb on the solid surfaces and depending on their surface charge they can cause either repulsion or attraction between solid particles. If the molecules adsorbed on the solid surface repel each other, the stability increase is observed. Regarding the situation in which the solid particles covered by the polymer or surfactant attract each other, aggregation and flocculation is observed. To sum up, the addition of surface active agents or macromolecules can cause either stabilization or flocculation of the described systems [8-9]. This is why methods that allow to study the stability of colloidal systems are very important. Due to continuous science development there are many 
available methods to study colloidal systems stability. They are UV-VIS spectroscopy, turbidimetry, zeta potential measurements, and density measurements [9-13].

\section{STABILITY}

Stability of the colloidal systems can be described by the Derjaguin, Landau, Verwey and Overbeek theory (DLVO) [14-15]. The abovementioned theory characterizes stability by the interaction energy of the particles in the solution [16]. The interactions between the particles can be divided into the van der Waals attraction forces and repulsive electric double layer forces. If the sum of the repulsion forces is higher than that of the attraction forces, the described system can be considered stable [17]. In other words when the attractive forces (caused by permanent dipole-permanent dipole interactions (Keesom), the permanent dipoleinduced dipole forces (Debye) and the transitory dipole-transitory dipole forces (London)) are stronger than the repulsive forces, the particles adhere with each other and as a result flocculation occurs [18]. The addition of different substances as well as other factors such as the concentration, ionic strength or $\mathrm{pH}$ can change the total potential energy of interactions [4]. In the case of the macromolecules addition the stability of the colloidal suspension depends on the mechanism of stabilization.

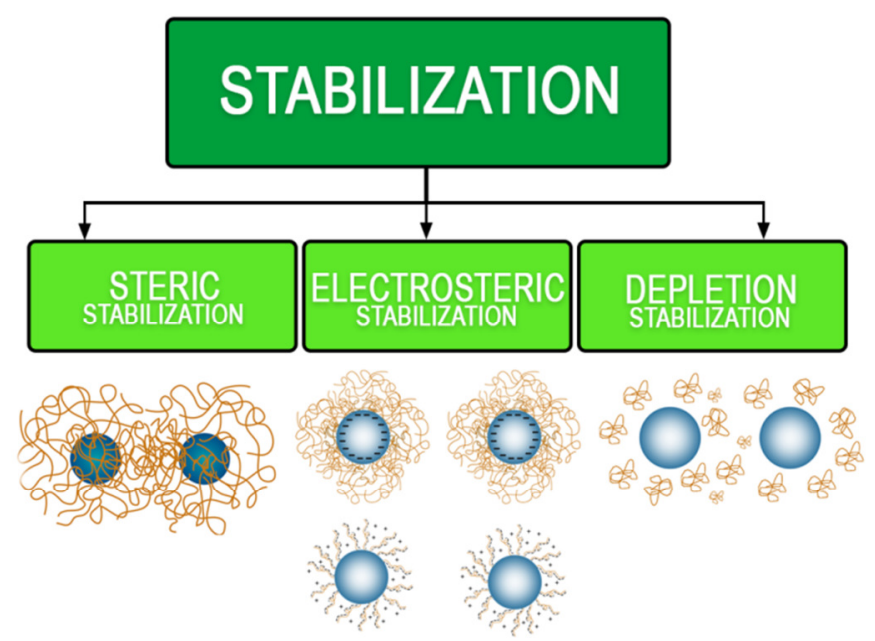

Fig. 1. Stabilization mechanisms caused by the addition of polymer to the colloidal systems. 
Different mechanisms of stabilization can be divided as follows: steric stabilization, electrosteric stabilization and depletion stabilization (Fig. 1) [19-21].

The steric and electrosteric stabilization mechanisms apply to the situation when the polymer is attached to the surface of the particle. In the case of the steric stabilization, the adsorbed polymer does not possess electrostatic charge. The electrosteric stabilization refers to the system in which the polymer has an ionic nature [19-20].

The last mechanism that is the depletion stabilization applies to the situation in which the polymer is not adsorbed on the solid particles. The free polymer chains, which can create different structures, are placed between the solid particles which leads to the attraction decrease between the particles and as a result, can influence the stability [21]. Although the addition of the macromolecule to the system might induce the stabilization increase, it might cause destabilization as well. The opposite of the stabilization process is the flocculation, which occurs when the sum of attraction forces are stronger than the repulsion ones [8]. Two main types of the flocculation caused by the polymer can be described. There are bridging and depletion flocculation (Fig.2).

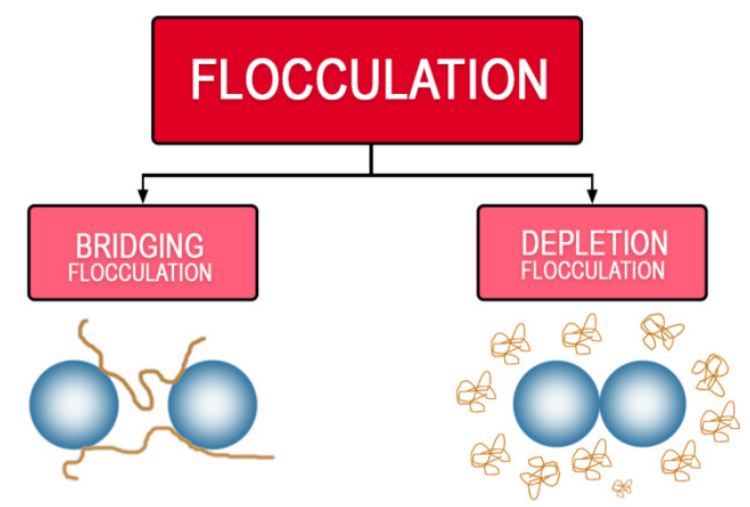

Fig. 2. Types of flocculation caused by the addition of polymer.

The bridging flocculation occurs when two or more colloidal particles are linked using polymer chains adsorbed on the solid surface, which results in the system aggregation. The depletion flocculation depends on the aggregation of solid particles influenced by some free unadsorbed macromolecules [21]. 


\section{METHODS USED TO DETERMINE THE STABILITY}

As it was mentioned before, different methods to characterize the stability of colloidal systems are used. The most common are the UV-VIS spectroscopy, turbidimetry, dynamic light scattering (zeta potential) and density measurements. The above-mentioned methods will be described in the next part of the paper.

\subsection{UV-VIS spectroscopy}

The UV-VIS spectroscopy method is based on the interaction of the light beam with the matter. When the light passes through the liquid solution, absorption, refraction and reflection of the light beam might occur. The principle of this method is the measurement of the light intensity before and after passing through the system. The ratio of the intensity of the passing light $\left(I_{t}\right)$ to that of the light before passing through the system $\left(I_{0}\right)$ is called the transmittance.

The absorption of electromagnetic radiation by the sample is described by the Labert-Beer equation:

$$
A=k \cdot c \cdot l
$$

where $A$ - absorbance, $k$ - the constant, $c$ - concentration of the solution, $l$ - thickness of the absorption layer.

Using the calibration curve method it is possible to determine the exact concentration of the component. The methodology is a bit different in the case of the colloidal stability measurements. In this case, the concentration is not important. The value of absorbance $(A)$ indicates whether the system is stable in time or not. To describe the stability of the colloidal system, such as the oxide suspension, before and after the addition of different substances, it is required to check the absorbance of the suspensions without the additives, and compare the obtained curves with those of the system after the addition of different molecules. If the system is more stable after the addition of the polymer, or another substance, the absorbance values will be higher and will decrease more slowly in time. Methodology in the case of the colloidal suspension can be described as follows: if the passing light beam encounters a solid particle on its way, it undergoes the process of refraction, reflection or absorption, which causes the absorbance change. As the measurement is 
performed in time, the absorbance values can be monitored throughout the experiment. The schematic methodology is shown in Fig. 3.
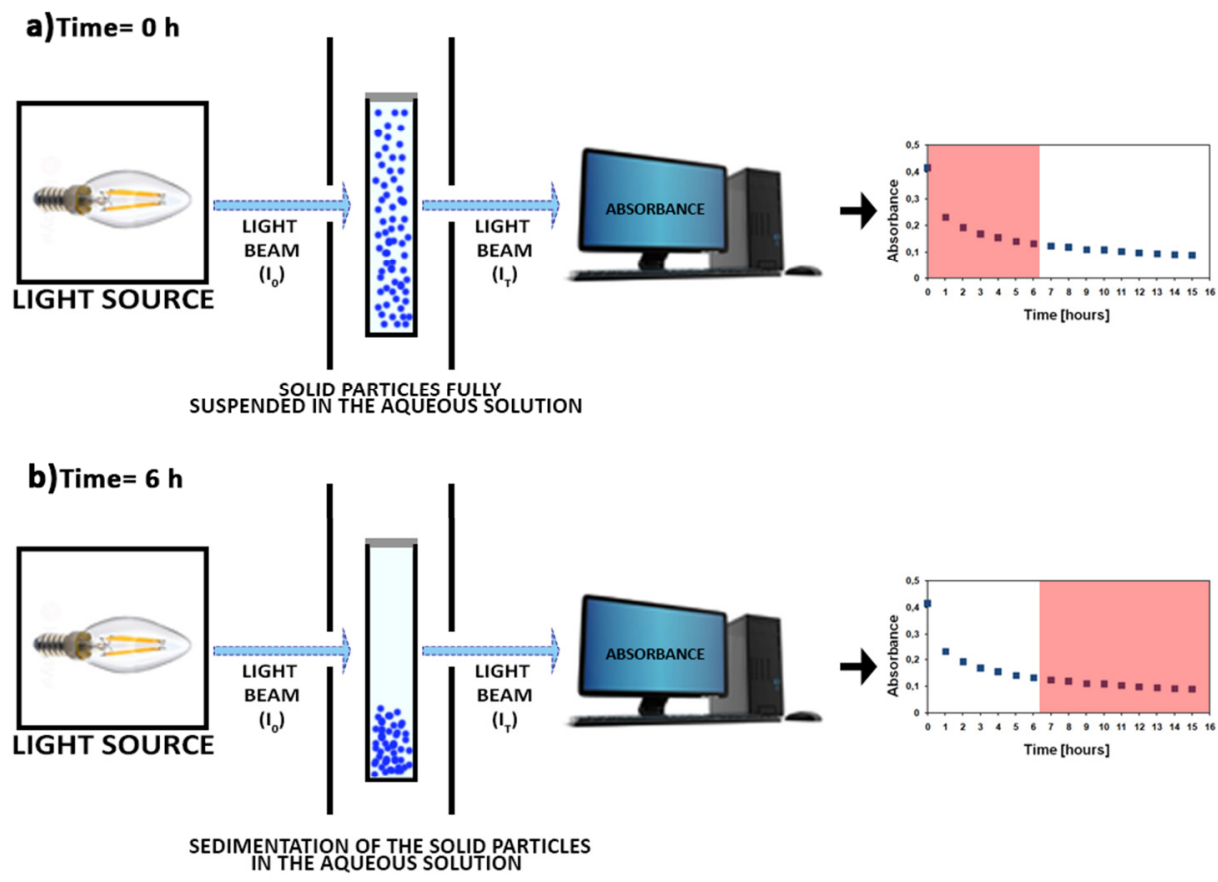

Fig. 3. Schematic representation of the measurement methodology using a UV-VIS spectrophotometer. The first instance (a) shows the situation where the prepared suspension is fresh and the sedimentation as well as flocculation do not occur. The second case (b) concerns the situation when the solid particles are flocculated and undergo the sedimentation process. The absorbance ranges corresponding to both situations are marked on the graph of the absorbance in time.

It is worth mentioning that the addition of polymer can not only cause stabilization, but also flocculation, which will be visible in absorbance values decrease. The exemplary graph of the stability measurement results obtained by using UV-VIS spectrophotometry is shown in Fig. 4. Two regions highlighted in different colours can be seen on the above-mentioned graph. The first one, starting from the start hour to about six hours shows a rapid decrease of the absorbance of both studied systems. After this time, from six hours to the last measurement performed within fifteenth hours, the absorbance values in the second region are almost the same. 


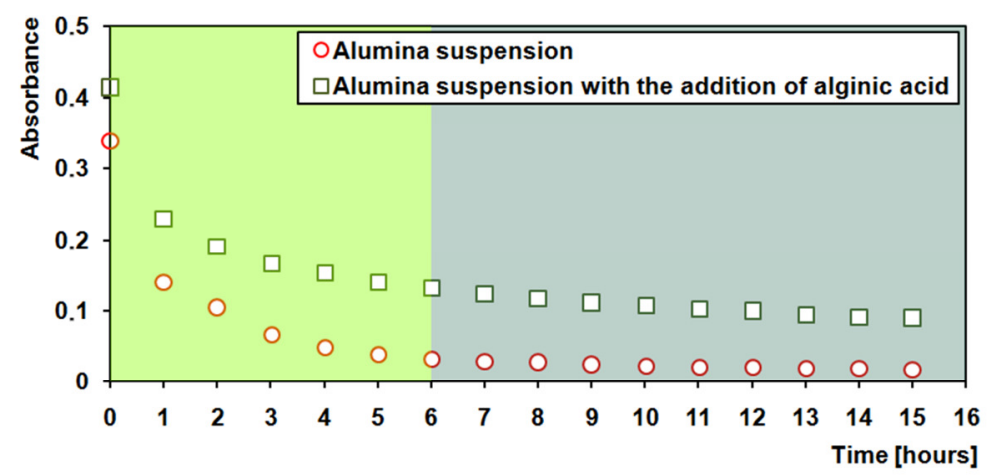

Fig. 4. Influence of the addition of alginic acid ( $\square$ ) on the stability of alumina suspension $(\mathrm{O})$, polymer concentration $\mathrm{c}=100 \mathrm{ppm}$, wave length $\mathrm{n}=550$ $\mathrm{nm}, \mathrm{pH}=5$.

The alumina suspension is considered unstable in time because the solid particles start to aggregate and flocculate due to the gravity force. However, after the addition of alginic acid the absorbance increase can be observed. This indicates that this polymer increases the alumina suspension stability. The main disadvantage of this method is the fact that the light beam passes thorough the suspension in one point and the obtained results refer to only this one point of the suspension. As a result, it is impossible to define where a clear layer or a sediment occurs.

\subsection{Turbidimetry}

The turbidimetric method is based on determination of the relation between the amount of light emitted by the source and the amount of light reaching the detector after passing through the tested colloidal system. The equipment that can be used in this type of measurements is the vertically scanning concentrated liquid dispersion analyzer which allows to document the changes in the particle size, for example caused by flocculation. Those changes are shown on the curve diagrams showing changes in the average particle size in time and the detailed analysis of the samples (determination of physical parameters such as migration speed, sediment thickness and determination of the turbiscan stability index- TSI) [8]. Technology of the device is based on the multiple scattering of light where the light intensity depends on the volume fraction and the average particle size of the dispersed phase. The instrument allows to analyze very concentrated dispersions (up to 95\%) with a wide particle size range (from $10 \mathrm{~nm}$ to $1 \mathrm{~mm}$ ). It consists of 
a head equipped with a light emitting diode (wavelength $880 \mathrm{~nm}$ ) and two synchronous detectors used for the examination of the transparent (transmittance) and concentrated (backscattering) dispersions (Fig. 5).

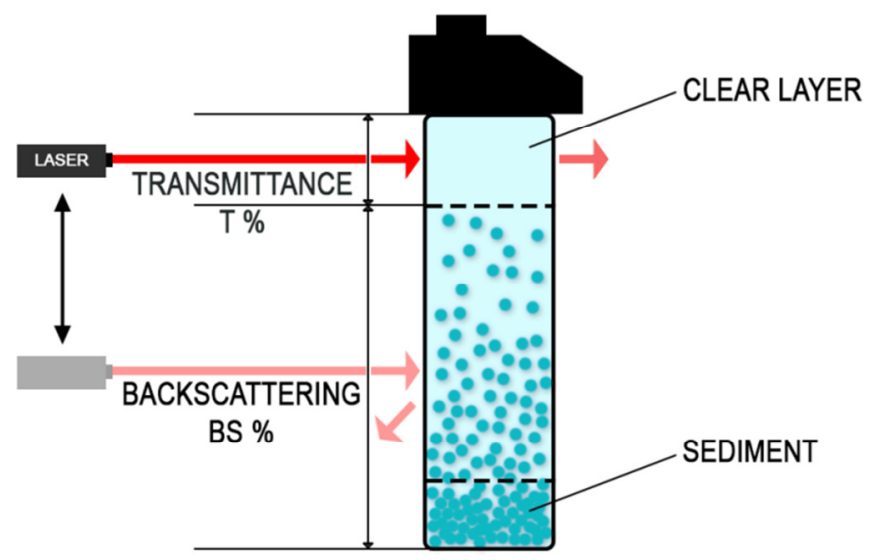

Fig. 5. Schematic presentation of the measurement conducted using the vertically scanning concentrated liquid dispersion analyzer.

The transmission detector records light passing through the probe at the angle $0^{\circ}$ in relation to the incident light direction. The other one is the backscattering detector registering the light scattered at the angle $135^{\circ}$. The great advantage of this device is that the head scans the entire sample vertically, which enables very accurate determination of stabilizingflocculating properties of the system, in comparison with other methods. The measurement results are presented in the form of curves showing the intensities of transmission and backscattering as a function of time. If the transmission for all samples is higher than $2 \%$, the stability parameter- the turbiscan stability index (TSI) is calculated from the transmission graphs. This parameter takes into account all single measurements during the experiments and the TSI value is obtained from their averaging. All processes taking place in the sample (including thickness of sediment and clear layer as well as particles settling) are summed up.

This parameter is calculated from the following formula:

$$
T S I=\sqrt{\frac{\sum_{i=1}^{n}\left(x_{i}-x_{B S}\right)^{2}}{n-1}}
$$

where: $x_{i}$ - the average backscattering for each minute of measurement, $x_{B S}$ - the average $x_{i}, n-$ the number of scans. 
The TSI values change in the range from 0 to 100 . The higher the TSI, the more unstable the system is [22].

\subsection{Zeta potential measurements}

Another method used to determine stability of various colloidal systems is the measurement of electrophoretic mobility which can be translated to the zeta potential. The zeta potential can be described as the electrokinetic potential measured in the slipping plane (Fig. 6).

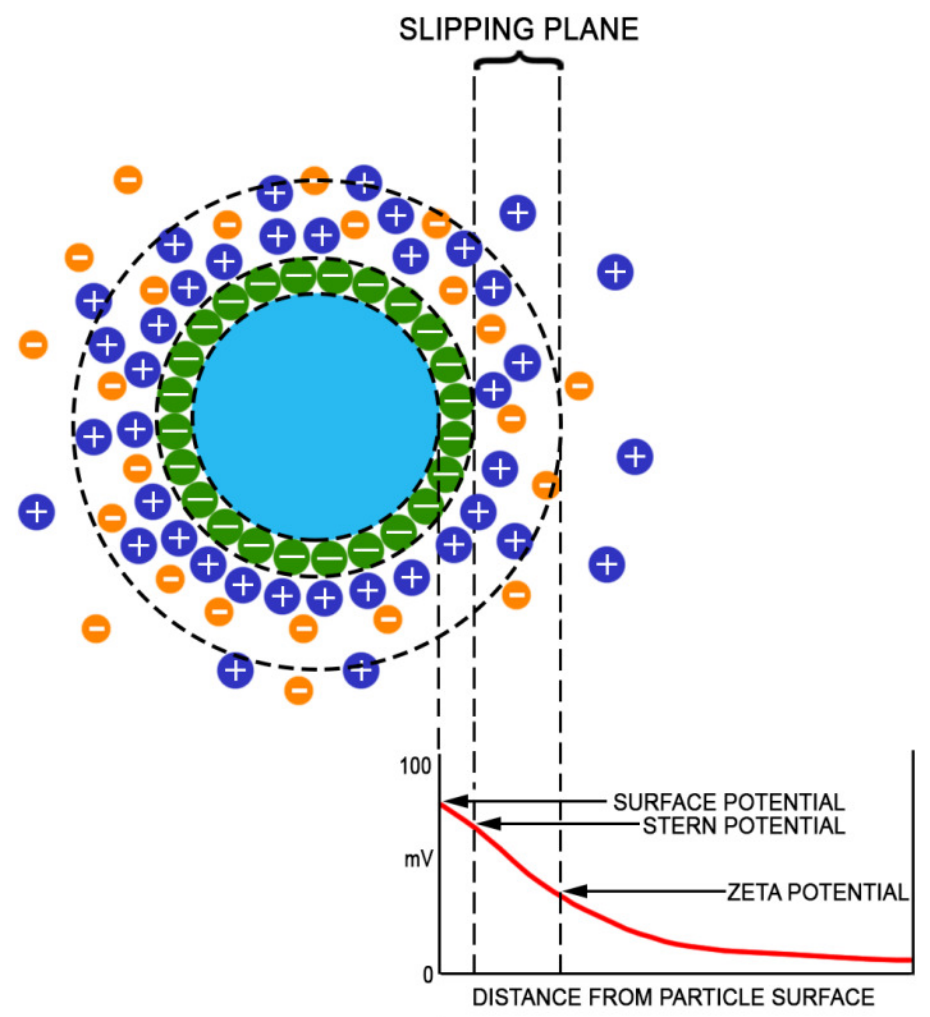

Fig. 6. The schematic view of the electrical double layer.

The more the slipping plane of the electric double layer is shifted towards the bulk solution, the smaller the zeta potential is. The addition of different macromolecules or ions to the solution pushes the slipping plane further, resulting in the decrease of the zeta potential. It is known that if the value of the zeta potential is higher than $\pm 30 \mathrm{mV}$, the system is considered completely stable [3, 23-24]. As it was mentioned earlier to maintain the stability of colloidal suspension, the repulsion forces have to be stronger than the attraction ones (Fig. 7). 


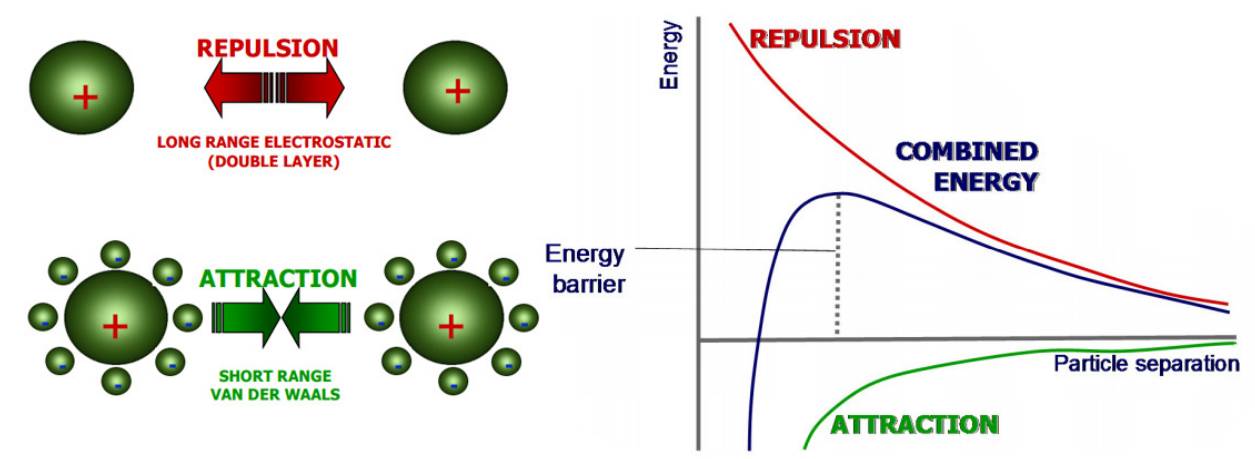

Fig. 7. Long range electrostatic repulsion forces and short range van der Waals attraction forces.

If the energy of the long range electrostatic repulsion forces is high enough, the Brownian motions occurs without the aggregation of solid particles. The measurement of the zeta potential is based on the electrophoretic mobility phenomena and can be obtained by measuring molecules velocity while they are moving due to the applied electric field. If a field is applied, particles and molecules will migrate towards an electrode. The technique used in most instruments is called electrophoretic light scattering (ELS). When the charged particles move back and forth in the applied electric field, changes in the intensity of the scattered light in accordance with the Doppler effect are observed [3]. Depending on the $\kappa_{a}$ parameter, the zeta potential can be calculated from three different equations which are Smoluchowski, Henry and Hückel equations. This parameter is described by the relation of the particle radius and to that of the electrical double layer. If the $\kappa_{a}$ parameter is larger than 100, which applies mostly to the large colloidal particles, the Smoluchowski equation should be used:

$$
u_{e}=\frac{\varepsilon \zeta}{\eta}
$$

where: $u_{e}$ is the electrophoretic mobility, $\varepsilon$ is the dielectric constant, $\zeta$ is the zeta potential and $\eta$ is the solution viscosity.

If the $\kappa_{a}$ parameter is between 0.1 and $100\left(0.1<\kappa_{\mathrm{a}}<100\right)$ the Henry equation should be used: 


$$
u_{e}=\frac{2 \varepsilon \zeta}{3 \eta} f\left(\kappa_{a}\right)
$$

where: $u_{e}$ is the electrophoretic mobility, $\varepsilon$ is the dielectric constant, $\zeta$ is the zeta potential, $\eta$ is the solution viscosity and the $\mathrm{f}\left(\kappa_{a}\right)$ is the function of $\kappa_{a}$ parameter.

Regarding the situation in which the particles are very small and the $\kappa_{\mathrm{a}}$ parameter is lower than $0.1\left(\kappa_{\mathrm{a}}<0.1\right)$, the parameter can be neglected and the Hückel equation should be used:

$$
u_{e}=\frac{2 \varepsilon \zeta}{3 \eta}
$$

where: $u_{e}$ is the electrophoretic mobility, $\varepsilon$ is the dielectric constant, $\zeta$ is the zeta potential and $\eta$ is the solution viscosity.

The schematic representation of the zeta potential measurements is shown in Fig. 8.

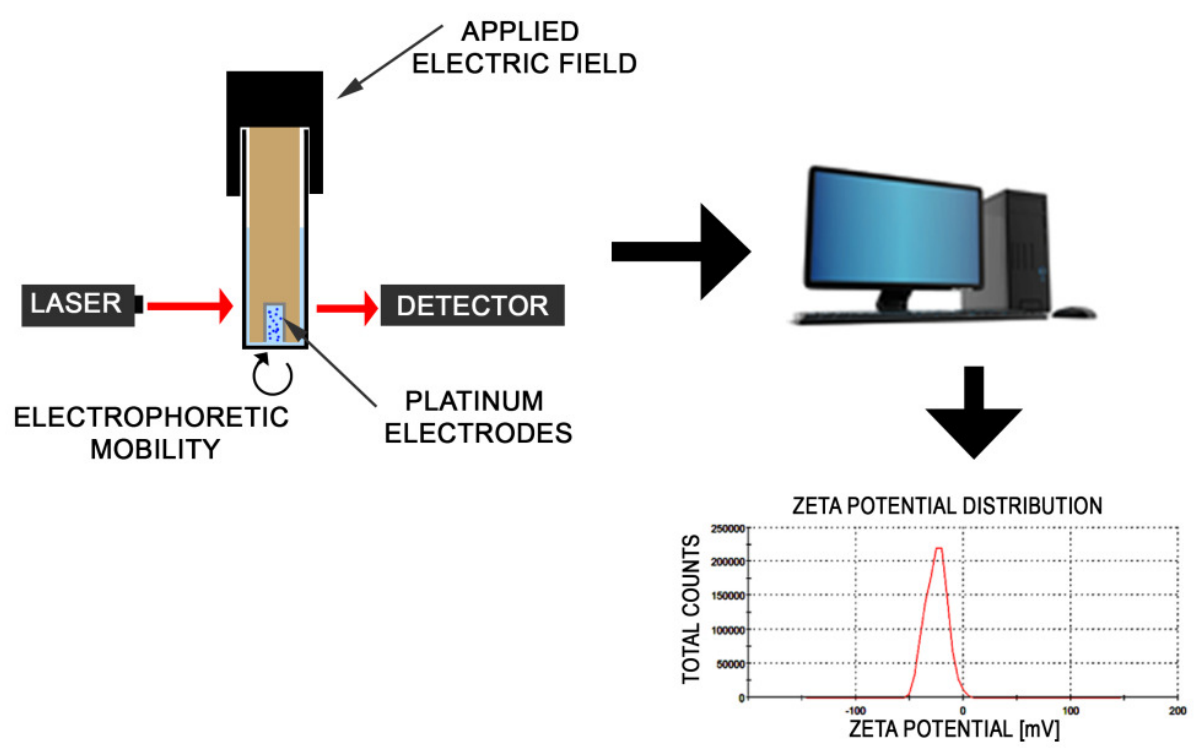

Fig. 8. Schematic representation of the zeta potential measurements in the dip cell. The applied electric field causes that the particles move towards the platinum electrodes, then the laser beam is scattered and the beam intensity is changed. The changes are registered by the computer and the zeta potential is calculated from the electrophoretic mobility using appropriate equation. 


\section{DENSITY MEASUREMENTS}

Another worth mentioning method used for determination of the colloidal systems stability is that developed by Pawlik et al. [25]. It applies to the systems in which the solid particle volume as well as the salt concentration are high. Such systems are opaque. To describe stability using this method densities of the supernatant, the pure dispersant and the suspension must be determined. After obtaining those values, it is possible to calculate the dispersion coefficient (DC) shown below:

$$
D C=\frac{\delta_{\text {sample }}-\delta_{\text {medium }}}{\delta_{\text {susp }}-\delta_{\text {medium }}} \cdot 100 \%
$$

where: $\delta_{\text {sample }}$ - the density of the supernatant, $\delta_{\text {medium }}$ - the density of the dispersant, $\delta_{\text {susp }}-$ the density of the suspension.

The higher the stability is, the higher the dispersion coefficient becomes. The value of DC $=100 \%$ means that the studied suspension is fully dispersed. If the DC value is 0 , the examined system is not stable. The undeniable advantage of this method is that it is very simple and expensive equipment is not required [25].

\section{CONCLUSIONS}

As it was mentioned earlier, the colloidal systems stability is essential in order to obtain the desired formulation. Ubiquity of such systems gives rise to development of some new methods used to investigate colloidal stability. All of the described methods are commonly used in different branches of industry.

\section{REFERENCES}

[1] E. Dickinson, „Colloids in Food: Ingredients, Structure, and Stability”, Annual Review of Food Science and Technology, Vol. 6, p. 211-233, 2015.

[2] P. Somasundran, B. Markovic, S. Krishnakumar, X. Yu, „Handbook of surface and colloid chemistry", CRC Press, , p. 127-192, Boca Raton, 1997. 
[3] V. Uskoković, Journal of Dispersion Science and Technology, 33, 1762-1786, (2012).

[4] E. Grządka, Colloid and Polymer Science, 293, 2845-2853, (2015).

[5] D.H. Napper, Polymeric stabilization of colloidal dispersions, Academic Press, London, 1983.

[6] P. Somasundaran, „Encyclopedia of surface and colloid science”, Taylor \& Francis, New York, 2006.

[7] E. Grządka, Cellulose, 18, 291-308, (2011).

[8] E. Grządka, Cellulose, 21, 1641-1654, (2014).

[9] M. Wiśniewska, K. Terpiłowski, S. Chibowski, T. Urban, V. Zarko, V.M. Gun'ko, Central Europen Journal of Chemistry, 11, 101-110, (2013).

[10] M. Wiśniewska, S. Chibowski, T. Urban, Reactive and Functional Polymers, 72, 791-798, (2012).

[11] M. Wiśniewska, S. Chibowski, T. Urban, Thin Solid Films, 520, 6158-6164, (2012).

[12] M. Wiśniewska, S. Chibowski, T. Urban, Adsorption, 16, 321-332, (2010).

[13] P. Somasundaran, X. Yu, S. Krishnakumar, Colloids and Surfaces, 133, 125-133, (1998).

[14] B.V. Derajaguin, L.D. Landau, Acta Physicochimica, 14, 633-662, (1941).

[15] E.J.W. Verwey, J.Th.G. Overbeek, „Theory of stability of lyophobic colloids", Elsevier, Amsterdam, 1948.

[16] J.L. Ortega-Vinuesa, A. Martin-Rodrigues, R. Hidalgo-Alvarez, Journal of Colloid and Interface Science, 184, 259-267, (1996).

[17] E. Grządka, Journal of Surfactants and Detergents, 18, 445-453, (2015).

[18] T. Sato, R. Ruch, ,Stabilization Of Colloidal Dispersion By Polymer Adsorption", Marcel Dekker Inc., New York, 1980.

[19] A.M. Sung, I. Piirma, Langmuir, 10, 1393-1398, (1994).

[20] R. Evnas, D.H. Napper (1973) Kolloid-Zeitschrift und Zeitschrift für Polymere, 251, 409-414, (1973).

[21] A.N. Semenov, A.A. Shvets, Soft Matter, 11, 8863-8878, (2015).

[22] TurbiscanLAB Expert brochure.

[23] I. Nurdin, MATEC Web of Conferences, 39, (2016).

[24] I.M. Tucker, J.C.W. Corbett, J. Fatkin, R.O. Jack, M. Kaszuba, B. MacCreath, F. McNeil-Watson, Current Opinion in Colloid \& Interface Science, 20, 215-226, (2015).

[25] M. Pawlik, J.S. Laskowski, A. Ansari, Journal of Colloid and Interface Science, 260, 251-258, (2003). 